\title{
Téoros
}

Revue de recherche en tourisme

\section{Le boom de la chirurgie à prix réduit}

\section{Laure Esnard}

Volume 24, numéro 3, automne 2005

Tourisme et santé, quelques perspectives

URI : https://id.erudit.org/iderudit/1071077ar

DOI : https://doi.org/10.7202/1071077ar

Aller au sommaire du numéro

Éditeur(s)

Université du Québec à Montréal

ISSN

0712-8657 (imprimé)

1923-2705 (numérique)

Découvrir la revue

Citer cet article

Esnard, L. (2005). Le boom de la chirurgie à prix réduit. Téoros, 24(3), 25-29. https://doi.org/10.7202/1071077ar d'utilisation que vous pouvez consulter en ligne.

https://apropos.erudit.org/fr/usagers/politique-dutilisation/ 


\section{Le boom de la chirurgie à prix réduit}

\section{Laure Esnard}

Le développement d'un tourisme à but médical fait couler beaucoup d'encre en Europe. Depuis plus d'un an, les principaux médias français ${ }^{1}$, aussi bien spécialisés que généralistes, se font régulièrement l'écho de cette nouvelle tendance, très en vogue car très économique. Et pour cause: la délocalisation des soins, car c'est ce dont il s'agit, est censée révolutionner les habitudes des Occidentaux en matière de loisir et de chirurgie. Après quelques échanges de courriels (tout se fait par Internet) et quelques heures de voyage, il est en effet possible de subir l'opération de son choix à moindres frais. Ce nouvel Eldorado fait grincer des dents les professionnels de la santé comme du tourisme, pendant que les cliniques et les agences de voyage "spécialisées" s'en frottent les mains!

II suffit de quelques clics de souris pour évaluer l'ampleur que prend ce phénomène. En tapant «tourisme médical » dans un moteur de recherche, un internaute peut facilement avoir accès à de véritables " offres commerciales ». Liftings et autres liposculptures sont ainsi vendus à grand renfort de publicité et, à première vue, en toute légalité, sur la toile. Pourtant, quand on se penche sur les différents codes de déontologie, aussi bien en médecine qu'en tourisme, force est de constater que de telles pratiques sont considérées comme répréhensibles. À la consultation des forums de discussion $^{2}$, on comprend vite pourquoi. En effet, les victimes existent et elles commencent à se faire connaître. Entre le paradis des soins à petits prix et l'enfer des ratages sans possibilité (!) de recours, il n'y a qu'un pas.

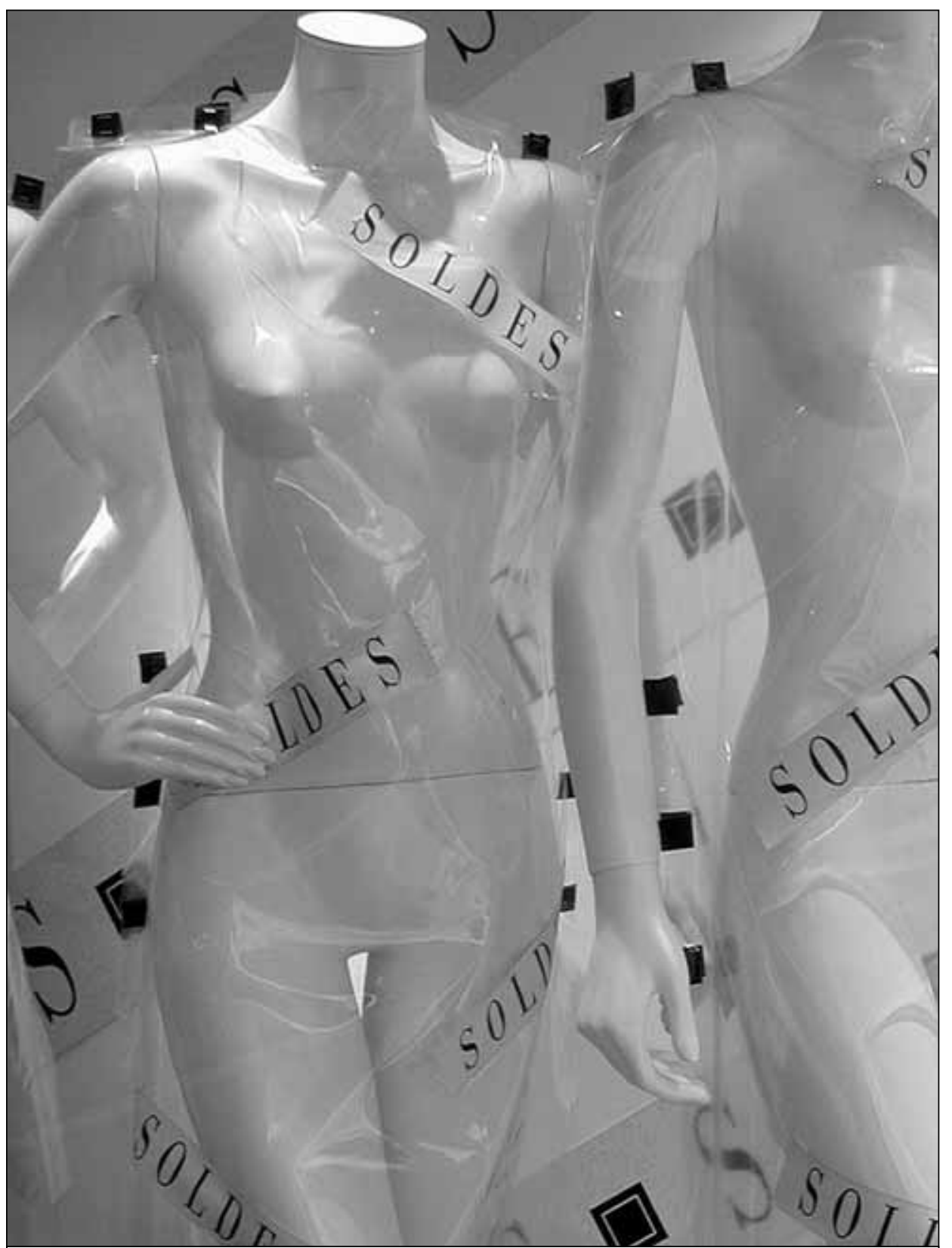

Soldes sur le Boulevard Haussmann, Paris.

Photo: @ Paris Tourist Office/Catherine Balet 
"Si vous vous trouvez sur ce site Web, c'est que vous êtes vraisemblablement en train de chercher une destination de vacances ou bien un organisme de chirurgie esthétique. " Dès la page d'accueil, le site de Beautiful Holidays ${ }^{3}$ annonce ses couleurs et propose de combiner ces deux possibilités. "Notre organisme est unique. Nous avons les moyens de vous trouver [...] un endroit de rêve où vous allez pouvoir vous détendre et vous remettre très rapidement de votre opération. » Sur Internet, les offres de ce type se multiplient. Elles proposent de joindre l'utile à l'agréable avec des forfaits qui comprennent le transport aérien, l'accueil à l'aéroport, les transferts, l'hébergement, des soins du corps et des excursions dans le désert ainsi que des chirurgies à la carte. Pour 3600 euros, on vous propose par exemple un lifting des seins! entre deux séances de sauna avec, en prime, cinq nuits dans un hôtel quatre étoiles ${ }^{4}$. À moins que vous ne préfériez opter pour des injections de Botox, une liposuccion de l'abdomen ou l'extraction d'une dent de sagesse. Et si vous avez besoin de plusieurs interventions successives, vous pouvez même bénéficier d'un forfait spécial. Tant qu'à se retrouver sur un lit d'hôpital, pourquoi ne pas profiter de l'occasion pour subir une deuxième opération dans la foulée? À ce prix-là, on ne compte pas.

En Pologne, les actes ophtalmologiques peuvent être jusqu'à $70 \%$ moins chers qu'au Danemark. Pour une implantation mammaire, une femme débourse autour de 9000 \$US aux États-Unis, alors que cette intervention ne lui coûtera que 2500 \$US en Afrique du Sud, safari en Land Rover compris. Loin d'être dispendieux, ces tarifs séduisent non seulement les Américains, mais également les Européens. Allemands, Autrichiens, Suisses, Italiens, Anglais et Français sont en effet de plus en plus nombreux à se laisser tenter par des hospitalisations grand standing à moindres frais. Rien n'est ni trop beau ni trop luxueux pour ces Occidentaux qui choisissent la Malaisie, la Thaillande ou encore les pays de l'Europe de l'Est comme lieu d'hospitalisation et les agences spécialisées dans ce créneau l'ont bien compris. Dès leur descente d'avion, elles conduisent leurs " clients $^{5}$ " en limousine jusqu'à leur chambre équipée de télévision par câble, d'air climatisé, de système de signal d'appel informatisé et de lits électriques. Certaines leur proposent même les services d'un interprète, le tout à des prix qui défient toute concurrence.

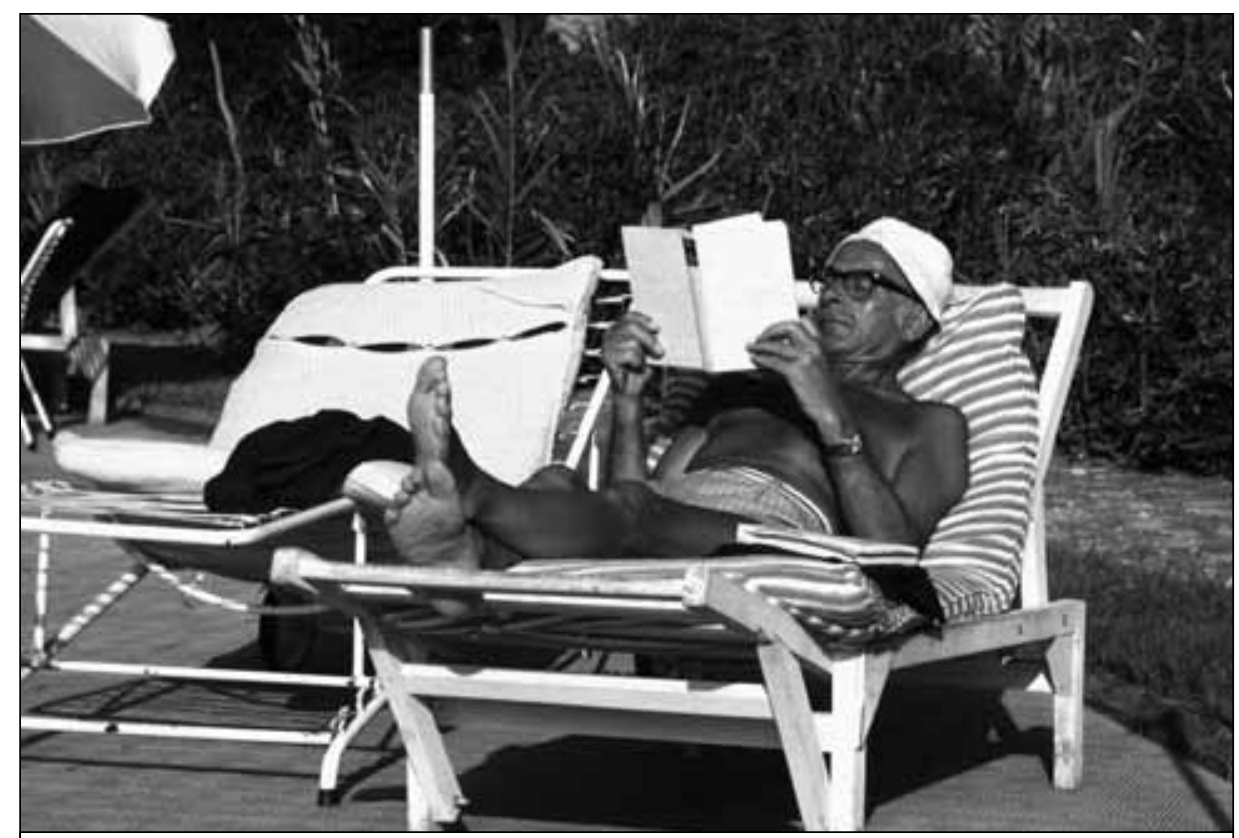

Vacancier à Tunis, Tunisie.

Photo : Alexis N. Vorontzoff/UNESCO

Des salaires locaux peu élevés permettent à ces établissements de proposer de tels tarifs, pour le moins compétitifs. On constate donc un engouement pour les formules dites «économiques » qui sont considérées comme une véritable aubaine en Asie du Sud-Est tout comme en Europe de l'Est et au Maghreb. Selon le Courrier du Vietnam, quotidien de langue française, la ferme de San Benito, aux Philippines, reçoit quotidiennement jusqu'à 80 clients venus des quatre coins du monde. Dans le Pacific Rim Magazine, on apprend que la clinique Bumrungrad de Bangkok accueille des patients canadiens par milliers. Sopron, petite ville frontalière située au pied des Alpes hongroises, compte de 150 à 400 dentistes, alors qu'il ne devrait pas y en avoir plus de 20. On y voit donc défiler, chaque fin de semaine, des milliers de personnes en mal de soins dentaires. En Inde, un million de touristes viennent se faire hospitaliser chaque année, contribuant ainsi, selon la Confédération d'étude indienne de l'industrie (CII), jusqu'à 5 milliards de dollars à l'économie du pays. Concession de terres, avantages fiscaux..., le gouvernement soutient le développement des hôpitaux privés depuis les années 1970. Résultat: ce pays est devenu la destination « idéale » pour les soins dentaires. L'État tunisien a lui aussi parié sur ce secteur touristique porteur. Aujourd'hui, il met un point d'honneur à encourager les investissements dans le tourisme médical en accordant des réduc- tions de taxes à l'importation du matériel. Des études prévoient déjà une explosion, dans les cinq prochaines années, du revenu généré par cette nouvelle activité au profit des pays dits en développement. Le Bureau philippin du tourisme estime par exemple à $25 \%$ la croissance annuelle de ces types d'établissements en Asie.

Les vacances chirurgicales semblent ne présenter que des avantages. Économiques et séduisantes, elles se veulent également sécuritaires. Histoire de rassurer les plus récalcitrants, la clinique thaillandaise Bumrungrad, par exemple, s'est bardée d'une série de références certifiant qu'elle répond à des normes de qualité rigoureuses (notamment ISO 9001-2000 et ISO 14001). Toutes les structures de soins disposent d'un équipement à la fine pointe de la technologie qu'elles mettent largement en avant dans leurs brochures publicitaires. L'agence malaise Beautiful Holidays affirme que ses docteurs et ses chirurgiens, formés aux quatre coins du monde, exercent "avec succès dans leur cabinet privé ». De renommée internationale, membres de l'académie scientifique de New York ou de la société américaine de chirurgie plastique, ils n'ont jamais fait l'objet de poursuites pénales et respectent des procédures chirurgicales fixées selon des standards internationaux. S'ils n'ont aucune raison de remettre en cause les compé- 
tences de ces spécialistes "triés sur le volet » et diplômés des plus prestigieuses universités, les plasticiens professionnels interrogés pour le besoin de cette enquête ne cautionnent pas pour autant les méthodes employées, surtout en ce qui concerne les pré-consultations à distance. Par courriel ou, dans le meilleur des cas, par l'intermédiaire d'une caméra numérique, cellesci sont souvent réduites au strict minimum.

Malgré le caractère sommaire des préconsultations, Houssem Ben Azouz, directeur de l'agence tunisienne Cosmetica Travel, assure que cette nouvelle médecine "discount " ne représente aucun danger. "Du fait de l'éloignement, les interventions ne sont réalisées que sur des individus bien portants. La santé physique et mentale est d'abord évaluée par le biais de questionnaires détaillés et d'entretiens téléphoniques approfondis. ${ }^{6}$ » Mais comment juger à distance de la faisabilité d'une intervention, de la motivation du patient, de la qualité de sa peau? Comment savoir si une culotte de cheval n'est pas due à un problème rénal ou de rétention d'eau? De simples entretiens téléphoniques suffisent-ils pour déceler de possibles dépendances (au tabac, à l'alcool, aux drogues...) ou détresses psychologiques? Car les psychologues sont unanimes sur ce point: le bistouri ne peut en aucun cas réparer les âmes... La plupart des agences recommandent vivement de consulter dans son pays d'origine avant d'entrer dans une clinique étrangère, mais aucune ne l'exige. Ces consultations en bonne et due forme sont facultatives et donc rarement comprises dans les forfaits. Au contraire, elles font généralement l'objet d'un supplément financier.

Quand on peut acheter un tee-shirt made in Thailand pour trois fois rien, personne ne se prive. Alors pourquoi tenir un raisonnement différent quand il s'agit de médecine? Parce que tout acte chirurgical, aussi bénin soit-il, comporte des risques, parfois mortels. Or, pour vendre leurs "produits », ces agences spécialisées qui proposent de faire disparaître ventre flasque, double menton et rides du front évitent de faire référence à toute notion de danger et de souffrance. II paraît indéniable qu'elles omettent de mettre l'accent sur certaines réalités, moins "vendeuses " que d'autres. En effet, si les forfaits préétablis promettent bains de soleil et autres excursions inédites, elles oublient de faire référence à d'éventuelles complications et effets secondai- res. À l'exception de quelques structures qui s'attachent à fournir une «information éclairée ", nombre de cliniques évitent de faire référence à d'éventuels problèmes tels rejet d'implant, changement sensoriel dans ou autour de la zone traitée, décoloration de la peau, nécrose des tissus, asymétrie, infection, évolution inattendue des cicatrices... Des effets secondaires qui peuvent être multiples et, surtout, qui pourraient survenir aussi bien après une hospitalisation en France ou à Montréal qu'à Bangkok. Soucieux de voir se banaliser cette nouvelle forme de tourisme et se multiplier ces agences «pas comme les autres ", les principales instances françaises concernées se sont mobilisées. Dans des communiqués de presse diffusés en juillet dernier, le ministère de la Santé ainsi que le Syndicat national des agences de voyage ont rappelé que

les actes médicaux de chirurgie esthétique ne relèvent pas du statut d'agent de voyages, défini aux articles L. 211-1 et suivants du code du tourisme qui disposent que les agences de voyage doivent se consacrer exclusivement à cette activité. Ainsi, une agence de voyage n'est pas autorisée à contribuer, de manière directe ou indirecte, par publicité ou information, à la vente de prestations de chirurgie esthétique et s'exposent au retrait de leur licence.

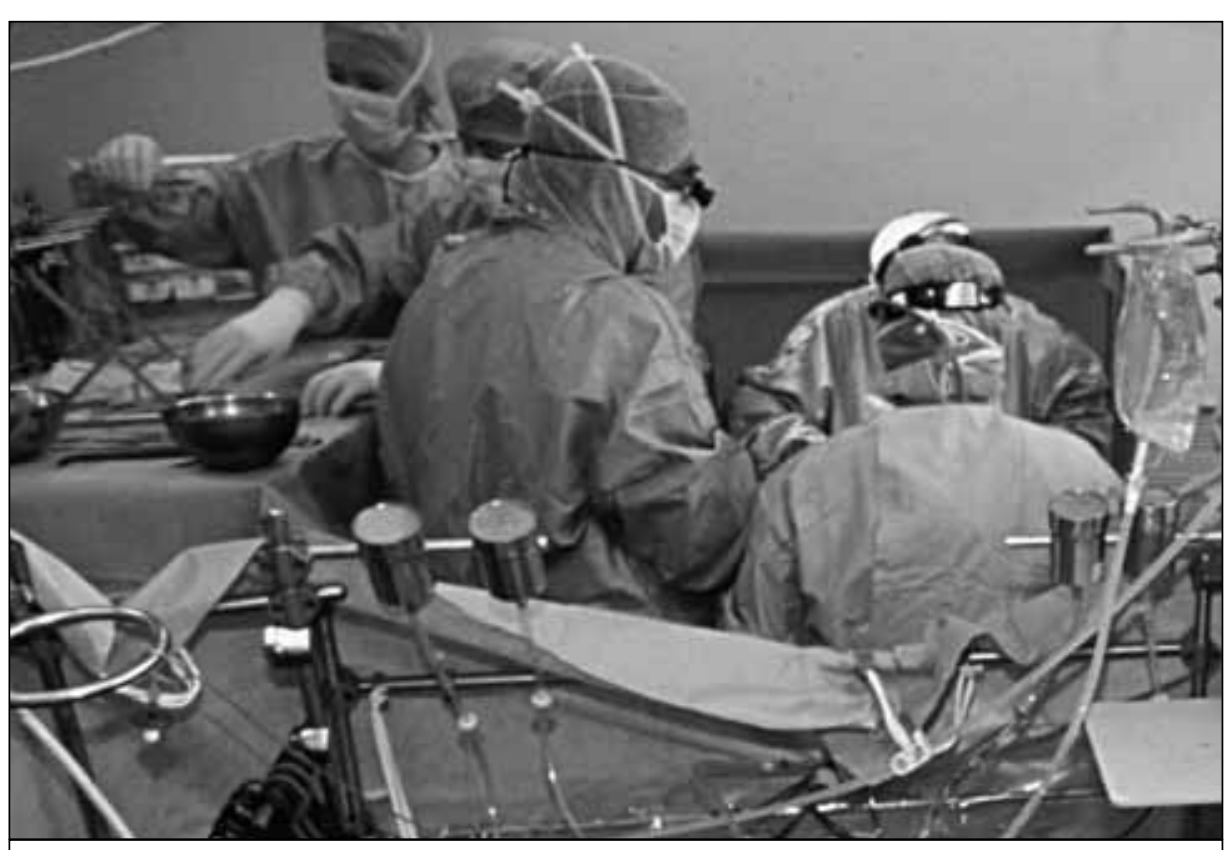

Salle d'opération, chirurgiens, matériel chirurgical et médical.

Photo : P. Lissac/O UNESCO
Si les risques liés à la chirurgie sont souvent éludés, les soins post-opératoires, eux, sont rarement abordés en détail. Les différents sites Internet ouvrent volontiers leur "livre d'or » pour mettre à la disposition de leurs visiteurs une multitude de témoignages de patients enchantés de leur expérience. Qualité des soins et de l'accueil, disponibilité du personnel, résultats impeccables... La méthode semble faire l'unanimité. Pourtant, les chiffres sont formels: d'après le Syndicat national français de chirurgie plastique reconstructive et esthétique, de $3 \%$ à $5 \%$ des interventions pratiquées sont des échecs au sens propre. Alors qu'en est-il de ces victimes ? D'abord, la majorité d'entre elles préfère souvent rester dans l'ombre car le traumatisme, selon les psychologues, peut s'apparenter à un viol. Le ratage est un tabou que l'on s'efforce de dissimuler aux yeux du monde. Bien sûr, les recours existent, encore faut-il pouvoir prouver les dommages causés. Certaines cliniques promettent qu'en cas de litige des arrangements à l'amiable sont toujours possibles. D'autres proposent au patient de choisir un plasticien «neutre» pour servir d'arbitre. Mais, quand de sérieux problèmes surviennent, les médecins peuvent se réfugier derrière l'argument de l'aléa thérapeutique et les attribuer à un événement incertain survenu à la suite d'une interventres effets, considérés comme impondération chirurgicale ou encore invoquer d'au- 
bles. Entamer une procédure dans ces conditions relève du parcours du combattant, surtout lorsqu'on se trouve à plusieurs heures d'avion de la clinique en question et que le litige relève de la compétence des tribunaux d'un pays étranger. Devant l'ampleur de la tâche et le coût des procédures, la majorité des victimes renonce à se lancer dans une bataille juridique.

Faute médicale établie ou accident malheureux, il reste une évidence: rien ne garantit un suivi sérieux. II arrive même que des cliniques étrangères désignent des généralistes comme médecins "référents " pour assurer les soins dans le pays d'origine des patients. Après leur séjour à l'étranger, certaines se plaignent de prothèses mammaires placées trop haut ou trop bas, d'autres se retrouvent avec un sein plus petit que l'autre sans qu'aucune solution sérieuse ne leur soit proposée. Des témoignages ${ }^{7}$, comme celui de cette française, opérée en Tunisie, alimentent les forums de discussion :

Quelques semaines après être rentrée chez moi, j'ai appelé la clinique pour me plaindre. Lorsque l'on m'a dit de m'adresser à mon omnipraticien pour régler mes problèmes, j'ai compris que les soidisant garanties de sécurité que m'avaient vantées les médecins n'étaient plus à l'ordre du jour et que l'on ne me proposerait aucune prise en charge gratuite. Surtout maintenant que j'étais à des milliers de kilomètres du lieu d'intervention. De toutes façons, les autorités de mon pays n'avaient aucun pouvoir de contrôle sur des pratiques réalisées en dehors du territoire national.

Devant l'urgence de la situation, une nouvelle opération doit parfois être envisagée dans les plus brefs délais. II n'est alors plus question de se lancer dans de longues tractations pour obtenir réparation. Et ce peut être le parcours du combattant pour trouver un spécialiste qui accepte de rectifier le tir. Car certains, comme ce dentiste français, refusent de le faire: "Je soigne les infections car c'est obligatoire. Mais je refuse de remettre les couronnes descellées si ce n'est pas moi qui les ai posées », déclare-t-il.

Jean-Luc Roffe, secrétaire général adjoint du Syndicat national français de chirurgie plastique reconstructive et esthétique, dénonce ceux qu'il qualifie de marchands de chirurgie:

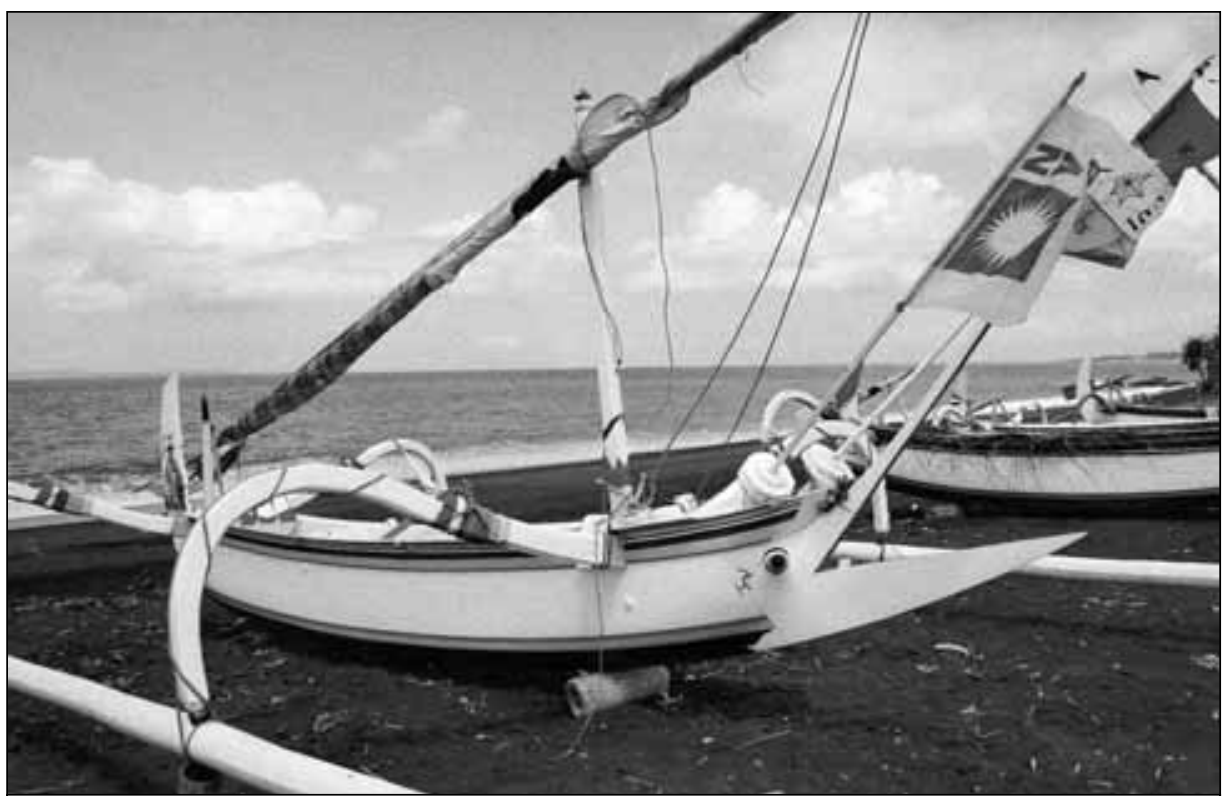

Bateaux de plaisance sur la plage, Bali.

Photo : Georges Malempré/O UNESCO

Ces cliniques n'ont aucun scrupule à violer les règles fondamentales de notre déontologie. II faut par exemple rappeler que selon le code français de la santé publique, la médecine ne doit pas être pratiquée comme un commerce. Le code stipule également que sont interdits tous procédés directs ou indirects de publicité et que la santé n'est pas un bien marchand. Quant à l'acte médical, il ne peut être considéré ni comme une denrée, ni comme une marchandise échangée pour une contrepartie financière. Si les praticiens français se comportaient comme ces marchands de chirurgie, ils auraient de sérieux problèmes avec la justice.

Et de citer le cas d'une jeune femme qui a dû se faire hospitaliser à ses frais pendant une semaine à raison de 1500 euros par jour (soit près de 2400 \$CA) à son retour de "vacances »: "En cherchant à faire des économies au détriment de sa sécurité, on s'expose au risque de devoir, finalement, débourser des sommes considérables ${ }^{8}$. " De nombreux spécialistes emboîtent le pas et affirment que ces situations sont engendrées par une pratique «douteuse » de la médecine basées sur une publicité mensongère. En faisant miroiter des bains de soleil sur la plage aux patients qui sortent d'une chirurgie, les tour opérateurs commencent à s'attirer les foudres de la profession, car « il faut rappeler qu'après une lipoaspiration par exemple, un grand pansement est appliqué sur la zone opérée. Celui-ci doit rester en place pendant environ huit jours, le temps minimum nécessaire à une cicatrisation débutante. Ni bain, ni douche ne sont autorisés pendant cette période, pas même dans la piscine d'un hôtel cinq étoiles ", souligne Jean-Luc Roffe.

Malgré les risques qu'elle induit, la chirurgie, associée aux notions de plaisir et de loisir est aujourd'hui commercialisée au même titre qu'une quelconque marchandise. Et ce commerce florissant s'appuie sur des techniques de vente imparables. Le patient, constamment entouré et rassuré, est l'objet de toutes les attentions. "Votre assistante personnelle vous prendra en charge dès votre arrivée à l'aéroport et vous suivra tout au long de votre séjour parmi nous » promet, par exemple, l'agence Estetika Tour. Une prise en charge de chaque instant qui ne laisse aucune place à l'hésitation pour que, une fois sur place, il ne vienne à l'esprit de personne de faire marche arrière. Devant le succès de cette médecine à prix réduit, les offres doivent également se plier à la loi du marché. Concurrence oblige, elles doivent se diversifier et rivaliser d'originalité. Après « tourisme et dialyse " et « tourisme et thalassothérapie ", l'AMI (Assistance médicale internationale) œuvre par exemple à la promotion de ce qu'elle considère comme son nouveau produit tunisien baptisé "tourisme et santé". Kaled Ahres, gérant d'AMI, propose des programmes personnalisés «adaptés au cas et aux désirs de chacun ", adaptant ainsi son offre à la demande. Pour susciter l'intérêt des Occidentaux en mal d'exotisme, d'autres 
misent sur des formules inédites basées sur des savoir-faire ancestraux. Pour retrouver ses vingt ans, pourquoi ne pas aller à Manille suivre un programme « détox-botox » basé sur des lavements de décoction de café combinés à des injections de toxine botulique? Ou sur l'île Maurice pour bénéficier de soins associés à la médecine (!) traditionnelle locale (massages, acupuncture...)?

Si certains se laissent séduire par les tarifs, les conditions proposées et les déclarations de victimes commencent à en rebuter plus d'un. En avril dernier, Arnaud ${ }^{9}$ pensait profiter de ses vacances pour se faire poser un pont. «Je me suis renseigné, mais j'ai vite abandonné cette idée. On m'avait conseillé une petite ville à la frontière autrichienne où l'on trouve davantage de dentistes que d'épiceries. Après réflexion, la barrière de la langue, l'absence de garanties et surtout de suivi m'ont découragé ", raconte ce Français qui, l'année dernière, a failli franchir le pas. Pour lui comme pour d'autres, ces nouvelles offres médicales intriguent d'abord, puis inquiètent. Les arguments présentés ne sont pas suffisamment convaincants et tout paraît si simple... trop simple, trop beau pour être vrai. Pour convertir les plus réfractaires et gagner en crédibilité, certains établissements adoptent une méthode différente. Ils choisissent de délocaliser les opérations en utilisant des méthodes davantage déontologiques et proposent des compromis rassurants pour tous ceux qui ne se sentent pas l'âme d'un kamikaze. Le centre suisse Label Esthétique se défend d'organiser des vacances chirurgicales, même s'il envoie ses patients se faire opérer en Tunisie. Avant de s'envoler pour l'Afrique du Nord, chaque candidat à la chirurgie doit rencontrer un spécialiste "en chair et en os ». Ces pré-consultations peuvent éventuellement être l'occasion de dissuader ceux qui espèrent des résultats irréalisables. Et, surprise, une fois sur place, il est clairement déconseillé de se lancer dans une visite de la région avec des cicatrices encore ouvertes: "On peut faire du tourisme, mais avant l'opération", est-il bien précisé sur leur site Internet. Le suivi post-opératoire, quant à lui, est bel et bien assuré dans une clinique de Lausanne. Des rendez-vous avec des spécialistes sont prévus jusqu'à six mois après l'intervention chirurgicale car, chez Label Esthétique, on considère que «le processus de cicatrisation n'est terminé qu'après un délai minimum d'un an ". Ces précautions paraissent indispensables, mais elles sont pourtant rarement mises en exergue.
Les actes médicaux qui ne font l'objet d'aucun remboursement sont désormais à la portée du grand public. Aujourd'hui démocratisée, la chirurgie n'est plus seulement au service de la santé, mais également de la beauté et de l'économie. Organisateurs de séjours all inclusive et praticiens indépendants profitent donc de cette tendance pour proposer une alternative aux soins dispensés en Europe et en Amérique du Nord et réaliser d'importants bénéfices, souvent au détriment du respect de règles fondamentales de déontologie médicale. Se faire refaire les cuisses, les dents ou la poitrine n'est plus un luxe, alors pourquoi s'en priver? Et, surtout, pourquoi s'en cacher? Car choisir un pays étranger pour se faire opérer, c'est aussi se mettre à l'abri des regards des amis et des voisins médisants, comme si gommer les effets de l'âge ou les imperfections du corps était un rêve inavouable qu'il faut réaliser en toute confidentialité. Et pour bénéficier d'une hospitalisation à moindre coût dans l'anonymat le plus complet, certains patients sont prêts à tous les sacrifices, comme à se laisser opérer par un chirurgien sans l'avoir jamais rencontré au préalable. Ainsi, partir en vacances en bonne mère de famille et en revenir avec la fraîcheur d'une jeune fille est aujourd'hui un rêve réalisable, qui peut également virer au cauchemar au moindre problème. Alors, to lift or not to lift, that is the question...

\section{Laure Esnard est journaliste.}

\section{Notes}

1 L'Express d'octobre 2005, le journal télévisé de TF1 du 17 mars 2005.

2 Se reporter, par exemple, au témoignage d'Ève sur le site Internet Vive les rondes, [www.vivelesrondes.com/forum/viewtopic_ 6105_15.htm].

3 [http://www.beautiful-holidays.com], consulté en juillet 2005].

4 Voir les promotions offertes à partir du lien suivant : [http://www.beautiful-holidays.com/page. asp?Languages $\mid \mathrm{D}=4 \&$ TitleReferencelD=113]

5 Entre guillemets parce qu'on devrait parler de patient; mais ces patients sont considérés comme des clients, consommateurs de chirurgie.

6 Propos recueillis lors d'un entretien téléphonique avec l'auteur.

7 Témoignages recueillis par l'auteur.

8 Propos recueillis lors d'un entretien téléphonique avec l'auteur.

9 Témoignage recueilli par l'auteur.

\section{Bibliographie}

Auteur inconnu (2005), « Philippines : lavements au café et légumes crus pour des stages détox ", Le Courrier du Vietnam, juillet, [http:// lecourrier.vnanet.vn/default.asp?CATEGORY ID $=14 \& N E W S P A P E R \_I D=54 \& T O P I C \_I D=65 \&$ REPLY_ID=26464].

Crick, Cynthia (2004), "Vacation in Ward B - Medical Tourism in Thailand ", Pacific Rim Magazine, [http://www.langara.bc.ca/prm/2004/ medical.html].

Des Déserts, Sophie (2005), "Le boom du tourisme médical ", Le Nouvel Observateur, n²113, mai, [http://www.nouvelobs.com/ articles/p2113/a267935.html].

Mommens, Françoise (2004), "Tourisme nouveau genre en Inde", Réseau de veille en tourisme, juillet, [http://www.veilletourisme. $\mathrm{ca} /$ fr/titre_resume_article.aspx?sortcode= 1.21.23\&id_article=225].

Saget, Estelle (2005), «Des pays très hospitaliers », L'Express, octobre, [http://www.lexpress.fr/ reussir/dossier/sante2/dossier.asp?ida=434541].

Schmidt, Florence (2004), "À l'Est, les nouvelles dents sont moins chères ", L'Hebdo, $n^{\circ} 40$, octobre, [http://www.hebdo.ch/index.cfm? id $=1165]$.

\section{Sites d'organismes organisateurs de séjours médicaux :}

http://www.beautiful-holidays.com

http://www.cosmeticatravel.com

http://www.estetikatour.com

http://www.bumrungrad.com

http://www.labelesthetique.com

http://www.apollohospitalgroup.com

http://www.travelsmart.net/ph/resorts/Batangas/ The_Farm_at_San_Benito/Welcome.html

\section{Sites Internet français consacrés à la santé}

http://www.plasticiens.org

http://www.e-sante.fr/fr/magazine_sante/mala dies_handicaps/Tourisme_medical_ne_vous _laissez_pas_tenter-8873-377-art.htm

\section{Sites Internet de professionnels du tourisme}

http://www.tourmag.com/Jean-DA-LUZ-redac tion-tourmag-com, Tourisme-de-sante-le-re mede-pire-que-le-mal-_a9015.html

http://www.quotidiendutourisme.com/index.php http://www.snav.org/ 